\title{
L'Adamawa, terre d'élevage
}

\author{
par A. MANDON
}

\section{I. - GÉographIE PHYSIQUe DU PAYS \\ Le Relief}

Au Centre du Cameroun français, entre le $6^{\mathbf{c}}$ et le 8 degré de latitude, l'Adamawa dessine un rectangle $d^{\prime}$ 'une superficie de $73.000 \mathrm{~km}^{2}$.

Bordée au Sud par la Sanaga et au Nord par la Benoué, adossée à l'Ouest au Cameroun Britannique, et à l'Est, à l'Oubangui-Chari, elle constitue une région charnière montagneuse qui opère la transition entre le Sud et le Nord-Cameroun.

C'est là, en effet, où vient mourir la forêt et naîlre la savane.

Peu accidentée dans sa partie méridionale, apparaissant comme une suite ininterrompue de croupes molles qui s'élèvent progressivement jusqu'au 7e degré de latitude, l'Adamawa forme autour de Ngaoundéré, chef-lieu administratif de cette région, une sorte de plateau central de 1.200 à 1.500 mètres d'altitude dont le socle, d'âye précambrien inférieur, est presque exclusivement constitué de granites noyés dans le basalte ancien et de gneiss, avec des formations volcaniques (récentes et anciennes) dans les régions surélevées.

Au Nord, elle se termine par une falaise abrupte qui surplombe la faille de la Benoué de près de 1.000 mètres.

Le plateau de l'Adamawa forme donc entre le Sud et le Nord du Territoire, une véritable barrière dont l'intérêt primordial est de protéger son cheptel contre les épizooties de peste et de péripneumonie qui se manifestent si fréquemment à l'extérieur de ses frontières.

Egalement, grâce à ses nombreux fleuves qui le parcourent en tous sens, ce plateau peut être considéré comme le plus important château d'eau du Cameroun puisqu'il alimente à la fois trois bassins différents, celui du Niger (par la Benoué et le Faro) du Tchad (par la Bini et la Vina) et de l'Océan (par le Djerem et le Lom).

\section{Ie Climat}

L'Adamawa est une région à climat subtropical. La Température moyenne, en corrélation avec l'altitude, s'avère relativement peu élevée : $22^{\circ} 05^{2}$ et varie entre $23^{\circ} 94$ en Avril et $21^{\circ} 07$ en Décembre.

L'Humidité relative est de 65 et passe de 41,8 en Fevrier, à 82,1 en Août.
Ouant à la Pluviométrie, elle est assez élevée : près de 1.600 millimètres par an, mais répartie en une seule saison des pluies qui va d'Avril à Octobre.

Au reste, voici de façon détaillée, l'évolution du temps dans le Secteur Adamawa (renseignements fournis par le Service Météorologique de Ngaoundéré).

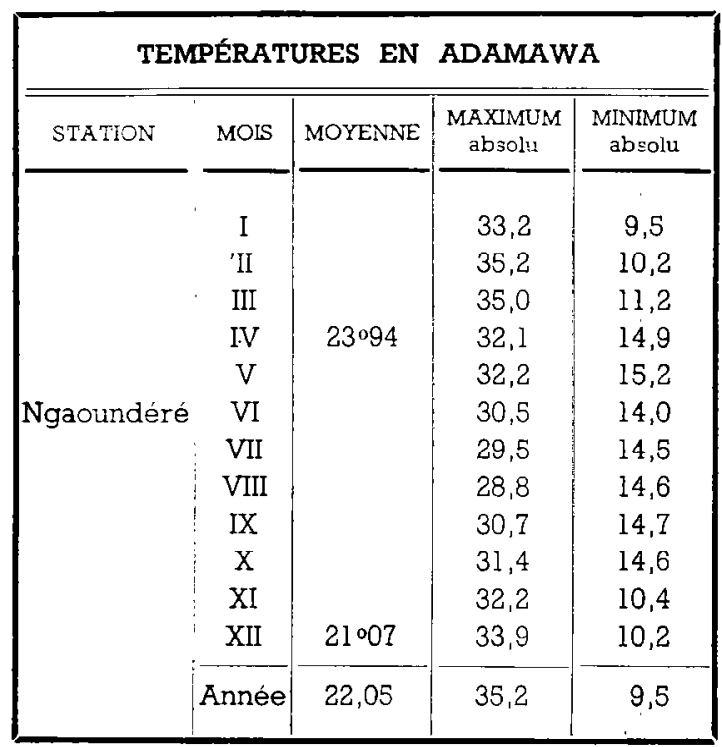

\begin{tabular}{|c|c|c|c|c|}
\hline \multicolumn{5}{|c|}{ CHUTES DE PLUIES } \\
\hline STATION & MOIS & $\begin{array}{l}\text { HAUTEUR } \\
\text { mənsuelie }\end{array}$ & $\begin{array}{l}\text { MAXIMA } \\
\text { en } 24 \mathrm{~h} \text {. }\end{array}$ & $\begin{array}{l}\text { NOMBRE } \\
\text { de jours }\end{array}$ \\
\hline \multirow{12}{*}{ Ngaoundéré } & $\mathrm{I}$ & 4,5 & 42,0 & 0 \\
\hline & II & 1,0 & 19,5 & 0 \\
\hline & III & 30,9 & 45,6 & 4 \\
\hline & IV & 152,9 & 70,8 & 13 \\
\hline & $\mathrm{V}$ & 198,2 & 79,2 & 19 \\
\hline & $\mathrm{VI}$ & 290,7 & 104,0 & $21^{s}$ \\
\hline & VII & 263,7 & 115,0 & 22 \\
\hline & VIII & 261,8 & 100,5 & 21 \\
\hline & IX & 253,1 & 80,3 & 21 \\
\hline & $\mathrm{X}$ & 153,7 & 48.5 & 15 \\
\hline & $\mathrm{XI}$ & 17,3 & 35,9 & 1 \\
\hline & XII & 4,3 & $84,0^{\prime}$ & 0 \\
\hline & Année & $1.631,6$ & 115,0 & 137 \\
\hline
\end{tabular}




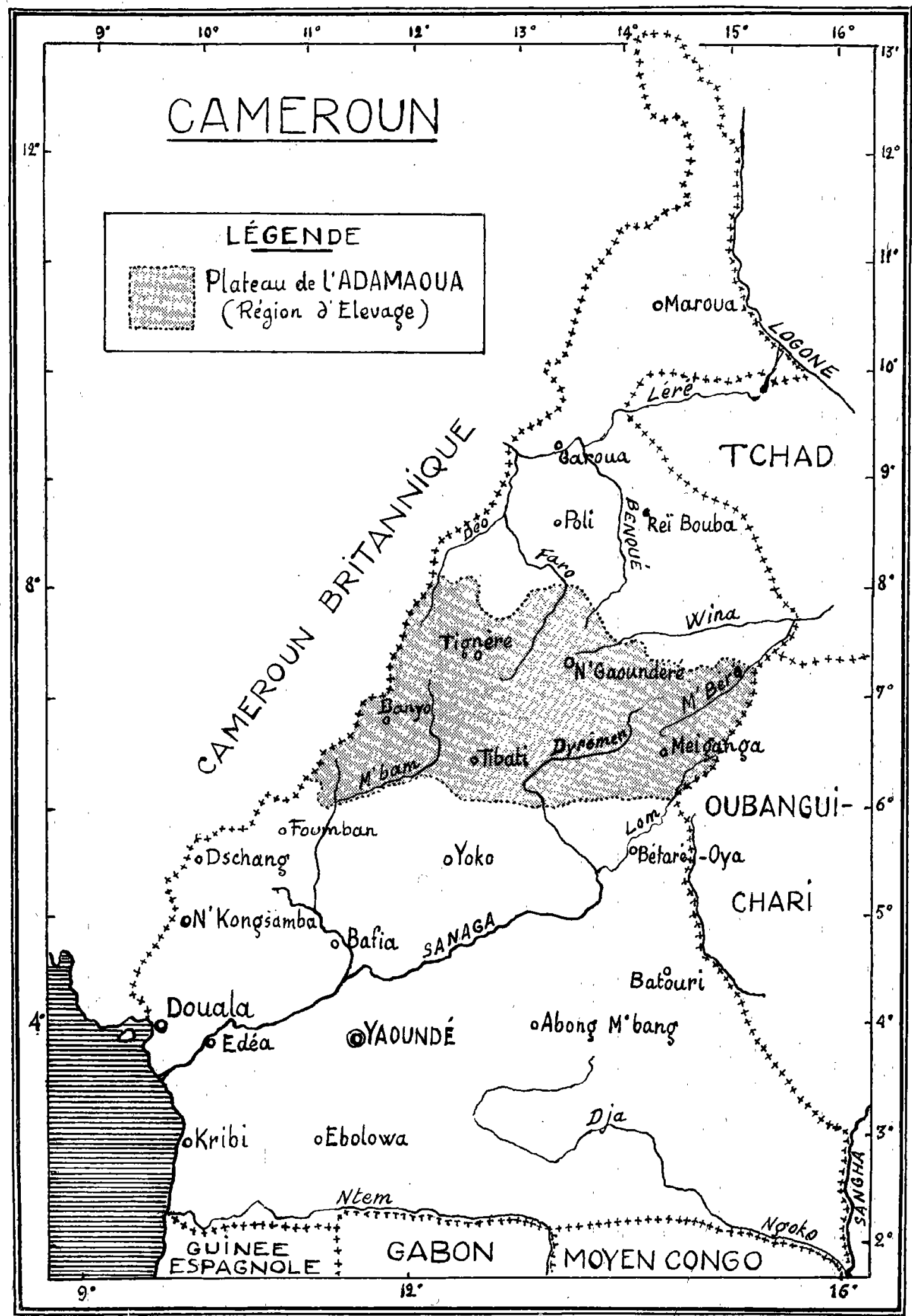


Source minérale. Lahoré de la Vina.

(Cliché Infocam)
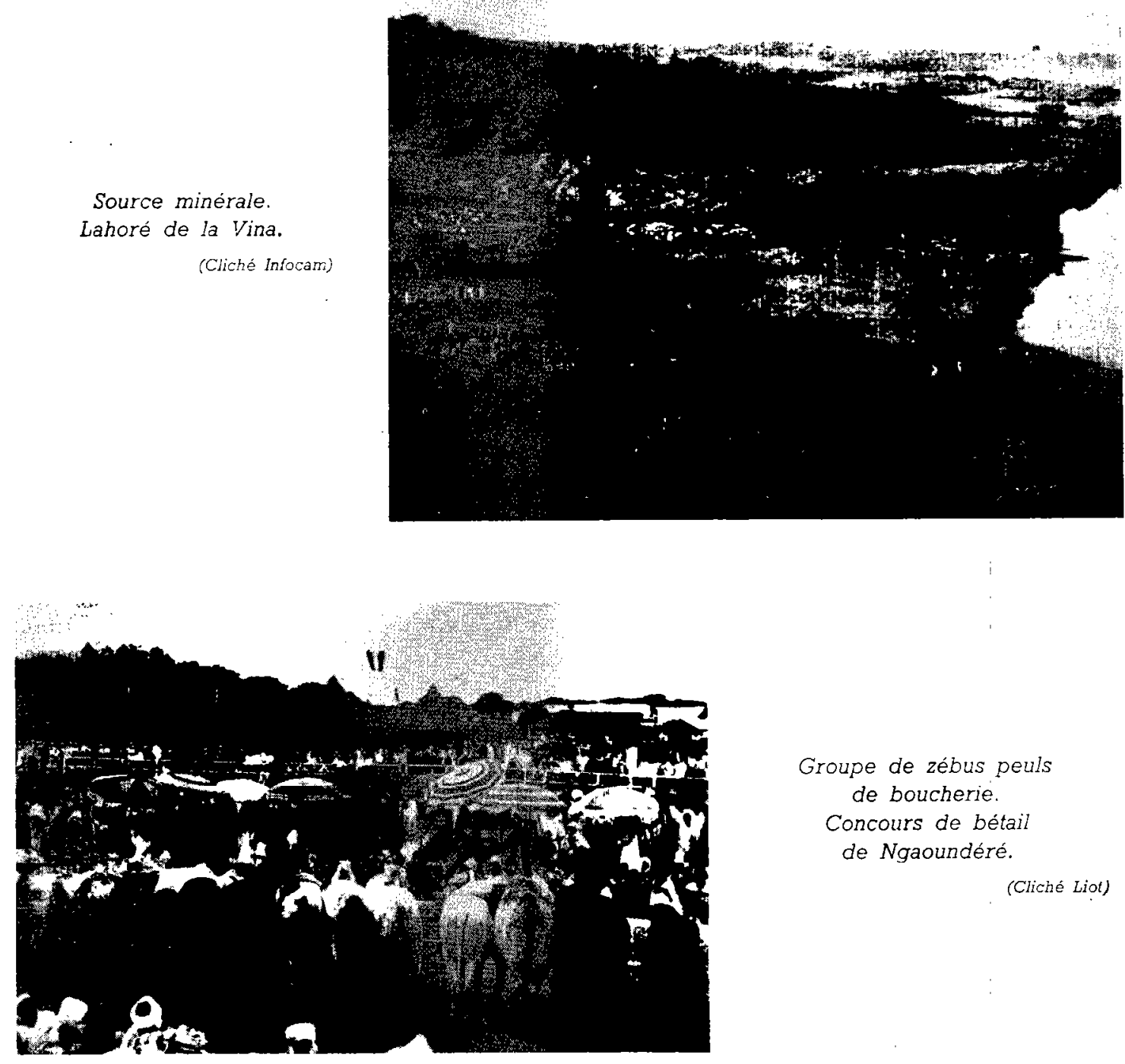

Groupe de zébus peuls de boucherie.

Concours de bétail de Ngaoundéré.

(Cliché Liot)
Eleveurs et bergers Mbororos et bétail en savane boisée.

(Cliché Tobie Schulier)

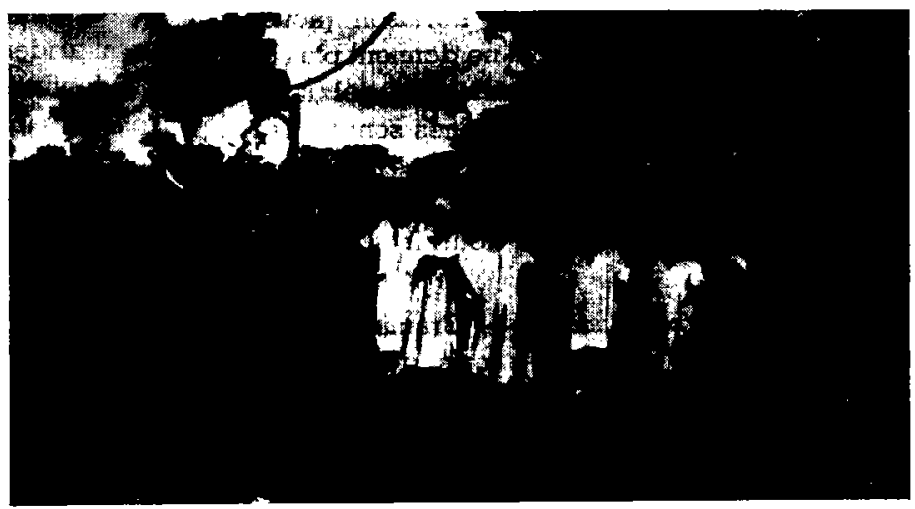


En conclusion, l'Adamawa est incontestablement la région à climat subtropical qui, par suite de l'altitude moyenne de ses plateaux, présente le climat le plus agréable et le plus voisin de celui des régions tempérées.

\section{Les Pâturages}

Le bétail de l'Adamawa se nourrit exclusivement d'un bout de l'année à l'autre de ce qu'il trouve sur les savanes herbeuses de ses plateaux. Jamais il ne reçoit de supplément de nourriture, même durant les cinq mois de saison sèche (de Novembre à Avril) où il transhume, soit dans les plaines basses asséchées (dénommées louggérés en langue peul) soit aux abords des innombrables cours d'eau qui irriguent le pays. Néanmoins, ce bétail se maintient bien. Il accuse même, sitôt la saison des pluies commencée, un état de santé florissant. Il faut donc admettre qu'il trouve sur les pacages qu'il parcourt les matériaux nutritifs nécessaires à son bon déveJoppement (1).

\section{Étude de la flore fourragère}

D'une manière générale les pâturages naturels de l'Adamawa sont constitués uniquement d'espèces fourragères appartenant à la famille des graminées.

Quant aux légumineuses, exception faite pour le Trifolium subrotundum que l'on rencontre en touffes clairsemées dans quelques terrains semi-arides, elles font ici totalement défaut.

\section{Principales plantes fourragères poussant naturellement en Adamawa}

La diagnose de ces plantes nous a été précisée par le Muséum d'Histoire Naturelle (Section Botanique) auquel nous avions adressé, il y a quelques mois, un herbier type des plantes fourragères recueillies par nos soins en Adamawa.

Pennisetum polystachyon. - Graminée annuelle de 1 mètre à $1 \mathrm{~m}$. 50 de hauteur poussant en touffes au début de la saison des pluies (mai-juin) dans les anciens terrains cultivés en mil ou en manioc.

Elle donne, lorsqu'elle est jeune, une herbe appréciée du bétail. Après la floraison (août-septembre) les tiges durcissent et ne donnent plus qu'un fourrage grossier. Elle disparaît au début de la saison sèche: néanmoins les graines sont facilement récoltées en novembre. Semée tardivement (juinjuillet) cette plante est susceptible d'être fauchée et fanée en novembre, et de fournir un excellent fourrage sec.

Pennisetum subangustum. - Graminée annuelle ressemblant à la première, mais de petite taille

(1) En Adamawa on estime qu'il faut de 4 à 5 hectares par tête de bovin.
(50 à 60 centimètres). Elle pousse en mai-juin dans les anciens terrains de cultures. Lorsqu'elle est jeune, elle donne une herbe tendre appréciée des animaux. Les graines apparaissent en novembre et sont faciles à récolter.

Disparition de la plante au début de novembre. Semée en juin, elle peut donner un excellent foin.

Chloris pycnothrix. - Graminée annuelle. Les tiges et les feuilles sont fines et rampantes. Elles atteignent cependant 30 à 40 centimètres de longueur. Bien acceptée du bétail local.

Eleusine indica gaertum. - Graminée également annuelle apparaissant en mai et juin au voisinage des abreuvoirs, des parcs à bétail el, en général, dans tous les lieux fortement fumés ou fréquentés par les troupeaux.

Les animaux l'apprécient lorsqu'elle est jeune.

Rhynchelytrum repens : Tricholaena rosea. Graminée annuelle qui pousse de mai a octobre, principalement sur les terrains anciennement travaillés. Les tiges sont semi-rampantes et peuvent atteindre 1 mètre à $1 \mathrm{~m} .50$ de haut. Feuilles très abondantes. La floraison commence en juillet et les graines sont faciles à récolter en octobre-novembre. Assurément, c'est l'une des meilleures graminées locales, très recherchée des bovins pendant toute la saison des pluies. Elle donne, en outre (à la Station Zootechnique de Wakwa) un foin d'excellente qualité.

Malheureusement les semis de graines réussissent difficilement.

Rottboellia exaltata forme glabra. - Graminée annuelle de grande taille qui pousse en grosses touffes, donnant des tiges grossières avec des feuilles rappelant celles du mil. Le bétail la consomme volontiers, mais la délaisse lorsqu'elle commence à se lignifier au début de la saison sèche.

Paspalum scrobiculatum commersonii. - Graminée vivace poussant toute l'année dans les marécages, où elle prend un grand développement, ainsi qu'en saison des pluies dans les pâturages humides et semi-arides. Elle donne des tiges denses de 30 à 40 centimètres de longueur avec des stolons qui forment un épais tapis végétal résistant au piétinement du bćtail.

Dans les pentes semi-arides, elle résiste à la sécheresse.

Le bétail l'apprécie fortement et la pâture en toutes saisons.

Sa propagation peut être faite par boutures ou par graines.

Sporobolus granularis. - Graminée vivace de 90 centimètres à 1 mètre de haut qui pousse sur les 
terrains semi-arides aussi bien en saison des pluies qu'en saison sèche. Les graines apparaissent en décembre. Tiges et feuilles sont consommées toute l'année par les animaux.

C'est une plante intéressante pour sa résistance à la sécheresse.

Sa propagation doit être faite par graines.

Sporobolus marginatus et pyramidalis. - Toutes deux sont appréciées du bétail, uniquement durant la saison des pluies.

Hyparrhenia rufa - Soluta violascens et chrysargea. - Graminées vivaces, résistantes à la sécheresse, prisées du bétail tant que les feuilles et les tiges ne sont pas devenues ligneuses.

Setaria pallidifusca et communis. - Graminée vivace de 40 à 50 centimètres de haut. Feuilles longues et fines très nourrissantes. Très bonne résistance à la sécheresse, durant laquelle la plante continue à donner de jeunes pousses vertes.

Beckaropsis uniseta. - Pousse par touffes dans les terrains arides. La végétation n'est pas arrêtée par la sécheresse, si bien que le bétail peut la consommer en toutes saisons.

Brachiaria stigmatisata. - Pousse également en touffes épaisses dans les terres arides, donnant sans interruption durant l'année entière des feuilles courtes et larges fort goûtées du bétail. Résiste bien au piétinement prolongé des animaux.

Brachiaria mutica. - Se développe dans les terrains humides ou marécageux et donne toute l'année des tiges de 60 centimètres de hauteur et de nombreux stolons.

Plante estimée du bétail.

Andropogon gayana. - Pousse dans les terrains arides et résiste admirablement bien à la chaleur. Plante excellente pour le bétail.

Pennisetum purpureum et violaceum. - Graminées vivaces, très communes dans tous les terrains légèrement humides. Poussent en touffes de 1 à 2 mètres et donnent des tiges grossières pourvues de nombreuses feuilles qui constituent un aliment de choix pour le bétail.

\section{Plantes fourragères introduites en Adamawa}

(Réserves d'élevage de la Station Zootechnique de Wakwa).

\section{Graminées}

Pennisetum clandestinum ou Kikuyu

Panicum Bardinode ou Herbe de Para

Paspalum dilatatum

Melinis minutiflora
Ces diverses graminées se sont parfaitement adaptées et rapidement propagées sur nos pâtures. Elles constituent pour nos troupeaux un aliment de valeur, dont la teneur moyenne en matières azotées est de 15,41. Néanmoins, si grande que puisse être la richesse nutritive de ces plantes, nous ne pensons pas, dans les circonstances actuelles, qu'il soit rentable de procéder à leur diffusion sur les pâturages " de brousse " de l'Adamawa.

\section{Legumineuses}

Indigofera. - Introduite ici sans succès.

\section{$* *$ \\ Les Iahorés ou sources minérales}

Doté d'un climat des plus cléments en raison de l'altitude, et riche de vastes étendues herbeuses dont la flore fourragère variée vient d'être précédemment étudiée, le plateau de l'Adamawa possède, en outre, le privilège de pouvoir mettre à la disposition de son cheptel de véritables sources minérales d'eau natronée appelées en langage peul : lahorés.

Captées. à proximité des rives des mayos et pourvues d'un système de pompage permettant l'aspiration ef le refoulement de l'eau dans des abreuvoirs, ces sources natronées, ou plus exactement d'eau chloro-bicarbonatée, sont fréquentées plusieurs fois l'an par des milliers et des milliers de bovins qui viennent s'y reminéraliser.

La plus importante, parmi les 15 qui sourdent en Adamawa est, à coup sûr, celle du Lahoré de la Vina, située a proximité de la station zootechnique de Wakwa et dont l'analyse des éléments dosés, effectuée par le Laboratoire de Nutrition appliquée de l'I.R.C.A.M. à Yaoundé, est la suivante :

Calcium : exprimé en mg

de ...............

Carbonates : exprimé en

$\mathrm{mg} \mathrm{de}, \ldots \ldots \ldots \ldots$

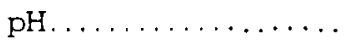

Alcalinite de titration. .

Chlorures. . . . . . . . . .

Magnésium ..........

Sodium . . . . . . . . . .

$\mathrm{CaO}$ par' litre $=185$

$\mathrm{CO}_{2}-\quad=2.793$

$-\quad=8,31$

$\mathrm{OCa}-\quad=1.568$

$\mathrm{CO}_{3} \mathrm{Ca}-=2.800$

$\mathrm{Cl} \mathrm{Na} \quad$ - $=3.660$

$\mathrm{MgO}-\quad=367$

$\mathrm{Na}-=810$

Faibles traces d'oligo-éléments tels que $\mathrm{Fe}-\mathrm{Cu}-$ Mn-I et F.

L'attirance du bétail pour ces eaux est donc due à la quantité élevée de CINa et de sels magnésiens. La saveur saline est perceptible au goût et si l'on ne peut parler de gourmandise quand il s'agit d'animaux venant se désaltérer, il est certain qu'il intervient un vieil instinct de besoin alimentaire 


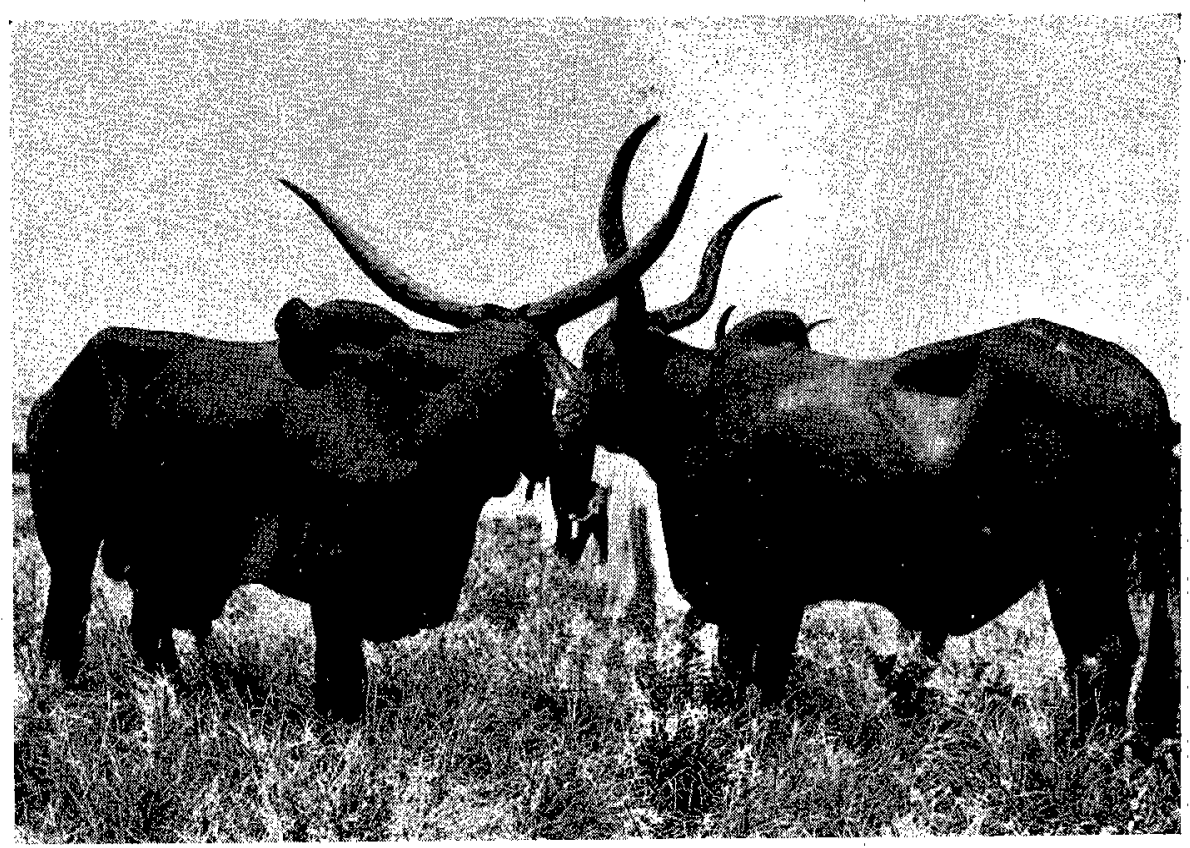

Zébus Mbororos de Boucherie.

(Cliché Mandon)

Taureau Mbororo, variété «Djafoun»,

7 ans, 620 kilos.

(Cliché Tobie-Schuller)
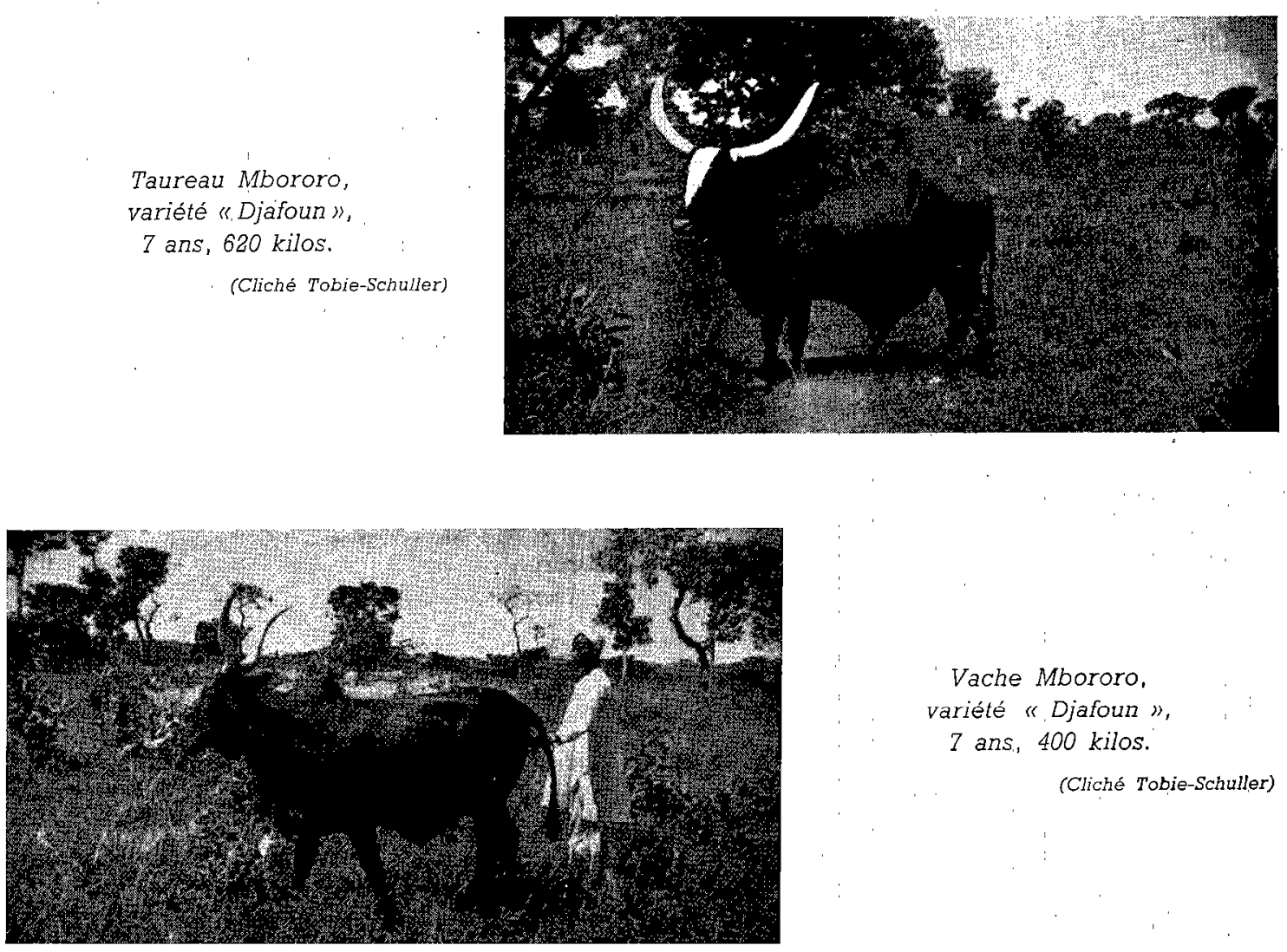

Vache Mbororo, variélé "Djafoun", 7 ans, 400 kilos.

(Cliché Tobie-Schuller) 
minéral concordant avec l'équilibre physiologique le plus satisfaisant de l'animal (1).

\section{II. - GÉOGRAPHIE hUMAINE HISTOIRE DE L'ADAMAWA}

Il y a environ 350 ans les Mboums, considérés aujourd'hui comme les premiers conquérants de la partie centrale du Cameroun, arrivèrent sur le plateau de l'Adamawa venant du. Nil, après avoir traversé la Nigeria. En réalité, ils pouvaient déjà, à l'époque, être tenus pour des conquérants car ils exterminerent les quelques humains qui y vivaient et dont la race n'a jamais été déterminéc.

Les Mboums, d'origine orientale et sans doute nilotiqué, étaient et sont demeurés des agriculteurs. L'élevage du bruf leur était completement inconnu.

A côté de cette population autochtone se fixaient plus tard, sans qu'on puisse en fixer la date, des groupes soudanais: Dourous, Koutines, Nyam-Nyam, Tikkar et Bayas, peup'es essentiellement chasseurs et agriculteurs.

A la fin du XVIII siecle, les Foulbés ou Peuls, tribu de pasteurs, sans doute originaire d'Asie, $\in \mathrm{n}$ tout cas de l'Egypte, pénétrèrent dans le NordCameroun, puis en Adamawa.

De taille moyenne, minces et droits, aux traits remarquablement fins, ils étaient alors essentiellement pasteurs, nomades et pacifiques. D'après certains auteurs, les Foulbés que les ethnologues disent être des Hamites, c'est-à-dire des éléments non nègres (si métissés qu'ils puissent être à l'heure actuelle) auraient déjà traversé lo Nord-Cameroun à une époque très ancienne, lors de leur migration initiale primitive de l'Est vers l'Ouest, et y auraient laissé sur place quelques élements, tels les Mbororos. C'est sans doute ce qui expliquerait pourquoi ceux-ci sont de type plus pur, ont mieux conservé les coutumes de la race et se sont laissés pénétrer moins profondément par l'Islam. Tandis que ce ne serait que vers le XVII siècle, au cours de leurs migrations de retour, de l'Ouest vers l'Est, que les Foulbés, noircis par leur long contact avec les populations négritiennes en Afrique Occidentale et profondément islamisés, auraient réapparu dans le Nord-Cameroun.

Quoi qu'il en soit, au début du XVIII ${ }^{e}$ siècle, plusieurs familles de pasteurs Foulbés se trouvaient déjà bien implantées dans le pays, et leurs troupeaux, au cours de leur transhumance, venaient même s'abreuver jusqu'au Lahoré actuel de la Vina.

Un peu plus tard, par une lente infiltration, maintes

(1) Rapport du Laboratoire de Nutrition appliquée de I'I.R.C.A.M. à Yaoundé (juin 1953). familles Foulbés, parmi lesquelles celles de Yillaga, $s$ 'installèrent définitivement sur les hautes terres, riches en pâturages auxquels ils devaient donner le nom de l'Adamawa (l) en souvenir du Modibo Adama, leur chef, lieutenant d'Ousman dan Fodio, fondateur, au début du XIX e siècle, du royaume de Sokoto et devenu chef de tous les Musulmans du Centre-Afrique.

A la pacifique nomadisation peule des premiers temps succéda, en effet, à l'appel à la guerre sainte lancé par le « Cheỉkou " Ousman, comme le célèbrent les Foulbés de l'Adamawa, un véritable soulèvement, qui devait donner aux Foulbés l'hégémonie dans cette région et aboutir vers 1835 à la' création de lamidats comme celuî de Ngaoundéré (2).

Les Mboums acceptèrent la suzeraineté des Foulbés. Quant aux Dourous, Koutines et autres peuplades païennes qui vivaient en Adamawa, lors de l'arrivée des Peuls, ils se soumirent ou bien échappèrent à leur domination jusqu'à l'arrivée des Allemands.

\section{Populations actuelles de l'Adamawa}

A. - Races d'origine hamitique :

10 Les Peuls ou Foulbés. - 83.270 habitants.

Ils ne constituent pas aujourd'hui une race pure en ce sens que l'administration considère, à tort, comme des Foulbés, un grand nombre de gens, d'origine locale, souvent indéterminée, le plus souvent des Mboums qui furent conquis par les envahisseurs foulbés et qui se métissèrent fortement entre eux.

Cette population islamisée est essentiellement d'économie pastorale.

$2^{\circ}$ Les Mbororos. - 10.250 habitants.

Ce sont les plus purs représentants de la race peule originelle. Restés à l'écart des autres races, et, en particulier des Foulbés, ils sont beaucoup moins marqués par l'Islam et se métissent fort peu... Leur caractère nomade s'atténue péu à peu, mais ils demeurent de véritables pasteurs.

Parmi les Mbororos de l'Adamawa, on distingue:

a) Les Wodabe venus du Bornou au commencement du XIXe siècle;

b) Les Djafouen venus de Djafoun (près de Kano);

c) Les Akoudji venus, semble-t-il, du Macina.

(1) Adamawa, en langue peule : l'endroit où se trouve Adama.

(2) Sociétés hierarchisées placées sous l'autorité d'un Lamido ou chef héréditaire entouré de ses " ministres ", dignitaires aux fonctions nettement définies et aux titres immuables.

Ngaoundéré : Montagne du nombril. La ville tire son nom de la montagne qui la domine et au sommet de laquelle se découpe une pierre: ayant la forme d'un puissant nombril. 
B. - Races d'origine soudanaise : 72.000 habitants.

Les Dourous, Mboums, Koutines et Nyam-Nyam sont les véritables populations autochtones conquises par les Foulbés et plus ou moins sérieusement islamisées. C'est surtout parmi les Koutines et les Nyam-Nyam que l'Islam a le moins pénétré. Les Bayas superficiellement islamisés sont venus d'A.E.F. Quant aux Baboutes, eux aussi islamisés, c'est un pcuple décadent, au passé cependant glorieux.

\section{C. - Races semi-Bantu : 10.000 habitants.}

Les Tikkars et les Kaka dont la plupart sont plus ou moins islamisés; les uns et les autres sont plus chasseurs qu'agriculteurs.

En général, toutes ces races soudanaises et semiBantu constituent aujourd'hui, en Adamawa, le noyau le plus homogene d'agriculteurs. Aucune ne s'adonne à l'élevage du Zébu.

\section{$*^{*} *$}

Comme on le voit, la population de l'Adamawa est très clairsemée ( 165.000 habitants au total), soit une densité de 2 habitants au kilomètre carré.

$\mathrm{Sa}$ situation démographique n'apparaît guère favorable. Chez les races islamisées, en particulier, le pourcentage d'enfants dépasse de peu celui des vieillards : $5 \%$ environ. Il y a là, pour les Foulbés, un problème crucial. La natalité chez eux continuant d'être des plus faibles et le personnel domestique dont ils disposent ne se renouvelant plus depuis que les razzia ont cessé en 1902, ils risquent de disparaître un jour de l'Adamawa.

Au reste, ce manque de main-d'œuvre explique la diminution progressive des superficies cultivées en mil (plus particulièrement sur le plateau de I'Adamawa) et surtout les difficultés que rencontrent aujourd'hui nos éleveurs noirs pour assurer le gardiennage de leurs 650.000 têtes de bétail.

\section{III. - GÉOGRAPHIE ANIMALE}

Avec les Peuls venus primitivement d'Egypte, puis du Soudan, apparut pour la première fois au XVIII siècle, sur le plateau de l'Adamawa, le bœuf à bosse ou Zébu.

En 1930, date des premiers recensements administratifs, l'effectif bovin de l'Adamawa etait numériquement évalué à 300.000 têtes. Aujourd'ui, il se chiffre à plus de 650.000 têtes : ce qui représente un capital cheptel d'une valeur très approximative de 5 milliards de francs C.F.A., soit 10 milliards de francs métropolitains.

C'est là à coup sûr, la plus importante richesse d'échange que possède l'Adamawa,

\section{Caractéristiques générales \\ du Zébu de l'Ádamawa}

\section{a) Type Zébu Peul}

Caractéristiques zootechniques.

La robe revêt des couleurs diverses, noire, blanche, froment, rouge et fauve. Les robes pie sont les plus fréquentes. Tête avec chanfrein long ct droit. Cornes relativement petites et à sections circulaires. Encolure assez étroite avec fanon développé. Bosse mi-musculaire, mi-graisseuse au niveau du muscle rhomboïde. Avant-main court et peu développé, fortement musclé chez le mâle. Arrièremain également court avec une croupe oblique et une cuisse souvent globuleuse.

En général ce sont des animaux d'un caractère docile et d'une rusticitć oxtraordinaire.

\section{b) Type Zébu-Mbororo.}

\section{Trois variétés:}

$1^{\circ}$ Le Djafoun, animal de très grande taille pouvant dépasser $1 \mathrm{~m}$. 60 en arrière de la bosse, et d'un tour de poitrine de $2 \mathrm{~m}$. 10 en moyenne, à robe acajou, au fanon pendant et très développé, aux cornes longues en forme de lyre, aux membres secs, puissants, et à la croupe fortement en pupitre. Poids moyen à 6 ans : 450 kilos.

$2^{\circ}$ L'Akou moins grand que, le précédent, d'une charpente osseuse moins puissante, moins grossière mais d'un format plus musclé.

La taille de l'Akou adulte varic, en 'effet, do $1 \mathrm{~m} .30$ a $1 \mathrm{~m} .40$ et son tour de poitrine de $2 \mathrm{~m} .10$ à $2 \mathrm{~m}$. 15. Quant à son poids, il peut atteindre à l'âge de 6 ans, de 500 à $550 \mathrm{kgs}$. C'est un zébu à robe toujours blanche (seuls le pourtour des yeux et des naseaux, ainsi que la face interne de la conque sont de couleur noire.) Ses cornes sont longues, en forme de $\mathrm{V}$ et le fanon très fourni.

$3^{\circ}$ Le Wodabe. - Intermédiaire entre le Djafoun et l'Akou. Peu rencontré aujourd'hui en Adamawa.

$$
\text { ** }
$$

Adaptés aux longues et incessantes randonnées à travers brousse, ces animaux sont d'instinct assez farouche, voire même agressif, mais comme les zébus peuls d'une très grande rusticité.

\section{Mode d'élevage}

L'élevage tel 'qu'il a toujours été pratiqué par les pasteurs Foulbés et Mbororos fixés en Adamawa, est de type essentiellement semi-nomade. 
6.

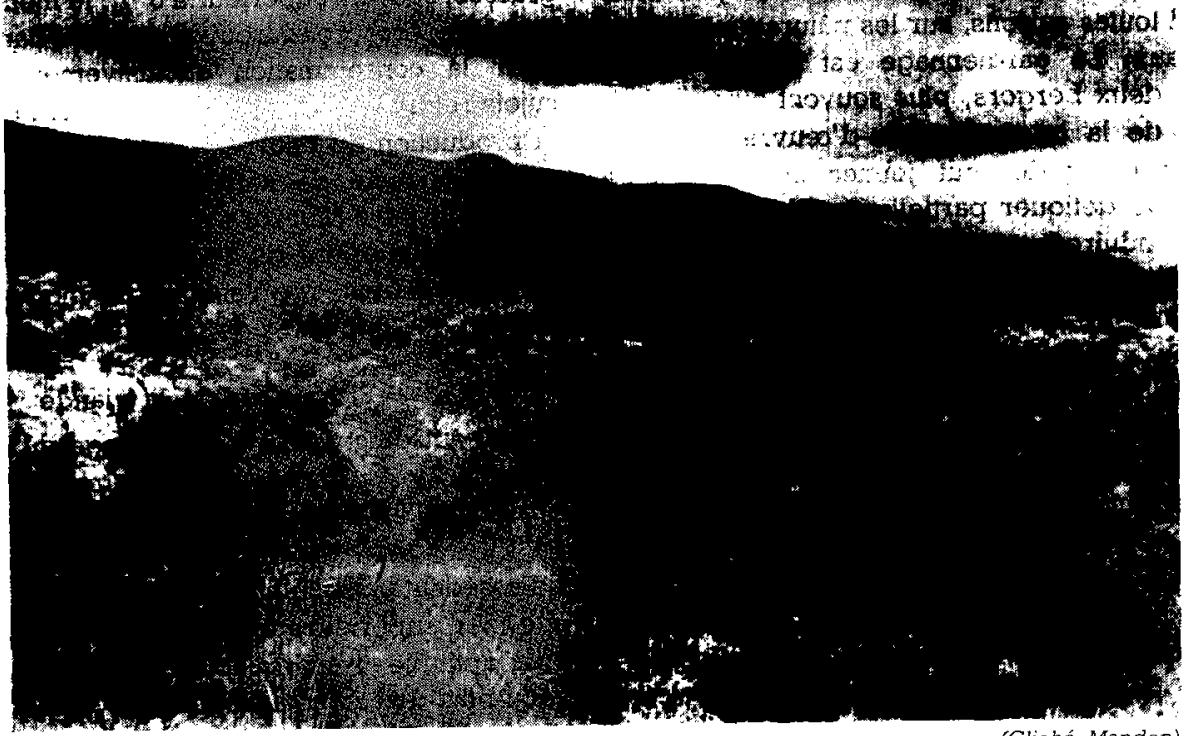

Idamawa, paysage type.

(Cliché Mandon)

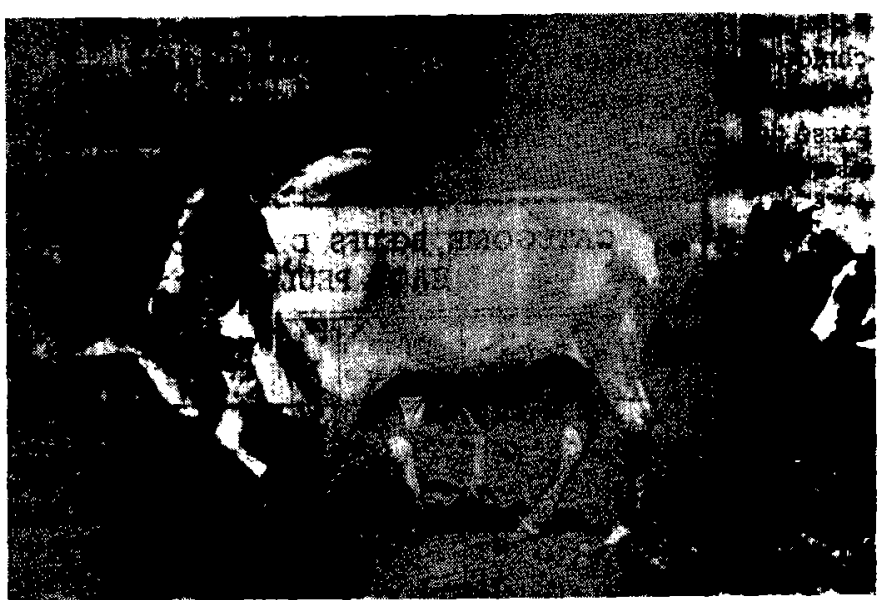

Génisse zébu peule, 3 ans.

(Cliché Mandon)
Troupeau de vaches peules au pâturage.

(Cliché Mandon)

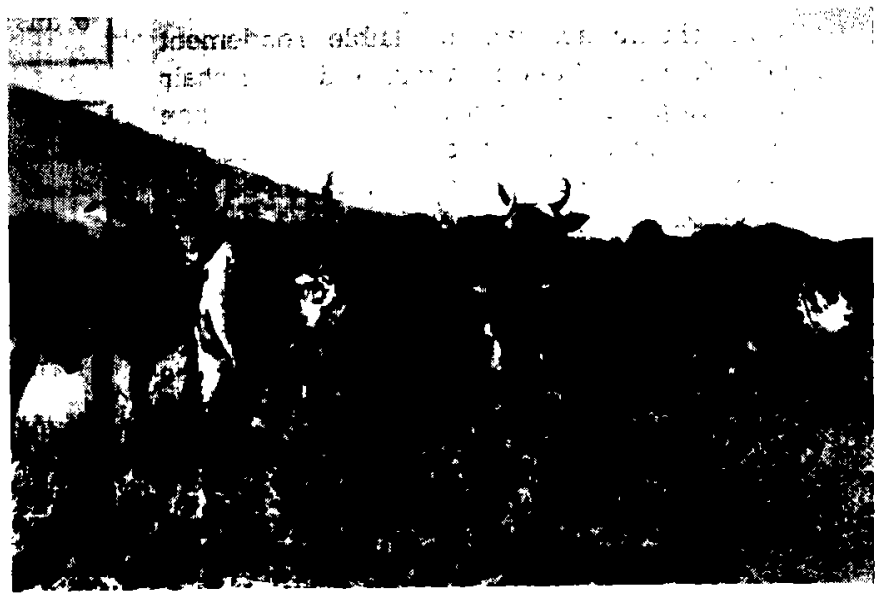


Les troupeaux, en effet, vivent nuit et jour sans abri et, en toutes saisons, sur les pâturages naturels des plateaux. Le gardiennage est parfois assuré par un ou deux bergers, plus souvent aujourd'hui, en raison de la crise de main-d'œuvre qui sévit en Adamawa par de tout jeunes enfants qui se conlentent de déliquer partiellenent leurs animaux et de les conduire quatre ou cinq fois l'an aux puits natronés de la région.

En général ces troupeaux se composent de 50 à 80 têtes au maximum, mais renferment une proportion élevée de taurillons, de taureaux et de vaches stériles ou âgées, près de $45 \%$ du troupeau. Il n'est donc pas étonnant, dans'ces conditions, que l'indice moyen de fécondité dans les troupeaux autochtones de femelles zébu Foulbés ou Mbororos soit relativement faible : 50 à $55 \%$, alors que ce même indice' chez les reproductrices zébues de la station Zootechnique de Wakwa atteint $88 \%$ (Rapport de gestion de la Station Zootechnique de Wakwa, juin 1953).

Egalement, on ne saurait s'étonner de ce que, faute de soins, le taux de mortalité chez les jeunes s'élève à 8 et $9 \%$ dans le troupeau foulbé au lieu de $2 \%$ à peine dans celui de notre Station Zootechnique.

Il est heureux néanmoins que l'effectif du troupeau bovin de l'Adamawa qui, de 1930 à 1953, est passé de 300.000 à plus de 650.000 têtes, soit un accroissement supérieur à $50 \%$ en 23 ans, n'ait été entravé depuis bien longtemps par aucune épizootie.

\section{UTILISATION ÉCONOMIQUE}

\section{Production du lait}

Les Zébus de race Mbororo (Djafoun-Akou et Wodabe) qui représentent à peine le quart de l'effectif bovin du troupeau de l'Adamawa, ne peuvent être considérés comme des animaux de boucherie. L'ossature extrêmement développée de ces animaux, leur manque total de précocité et d'aptitude à prendre de la graisse, font que ces grands marcheurs accusent un faible rendement en viande ( 40 à $42 \%$ ) et que la qualité de leur chair n'est que peu prisée du consommateur. L'utilisation de ce bétail pour la boucherie, et plus encore sorı exportation vers les centres consommateurs se sont révélées depuis longtemps n'être pas en Adamawa des opérations bien rentables.

Aussi, comme depuis toujours, le Mbororo à l'inverse du Peul, pratique systématiquement la traite sur ses femelles zébu Mbororos, dont les effets néfastes se font, hélas, habituellement sentir sur le développement des jeuncs à la mamelle, et comme le lait recueilli ( 300 litres au maximum par animal pendant les 6 ou 7 -mois que dure la lactation) renferme un taux très élevé de matières grasses, souvent supérieur à $6 \%$, le meilleur revenu du bétail Mbororo se trouve donc être dans l'abattage pour la consommation exclusivement locale des sujets réformés, et surtout dans la vente du lait pour la production du beurre.

La Beurrerie de Meiganga aménagée au cœur même du pays Mbororo peut ainsi évacuer anıluellement à l'interieur du Territoire de 50 à 60 tonnes de beurre frais, représentant un mouvement commercial de 20 millions de francs C.F.A. par an.

\section{Production de la viande}

Tout autre est l'objet de l'élevage du bétail zébupeul sur les plateaux de l'Adamawa. La fonction essentielle de cet animal est en effet la production de la viande.

Possédant un squelette des plus légers, une précocité bien supérieure à celle du Mbororo, et surtout une aptitude remarquable à l'engraissement, le zébu-peul de l'Adamawa constitue, aux dires mêmes des spécialistes, le meilleur bétail de boucherie de l'Afrique Noire.

Voici à titre indicatif les poids et les rendements moyens enregistrés sur les 300 bovins de type zébu-peul présentés en novembre 1952, par les éleveurs foulbés eux-mêmes, au Comice Agricole de Ngaoundéré.

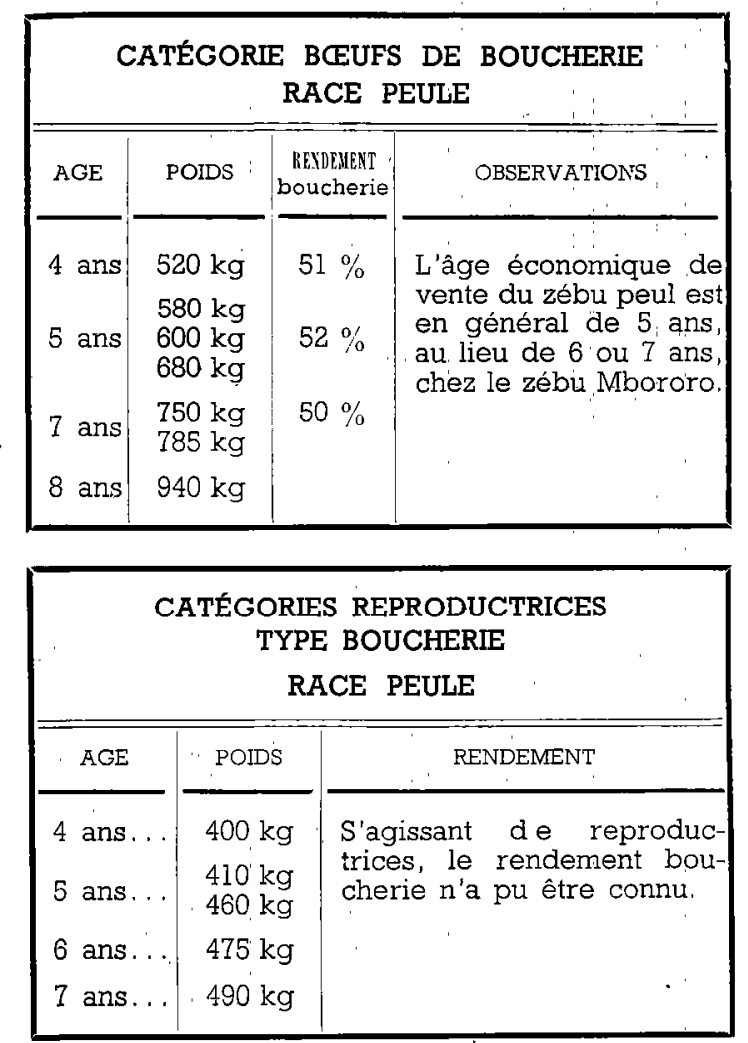


Taureau race peule.

(Cliché Ljot)

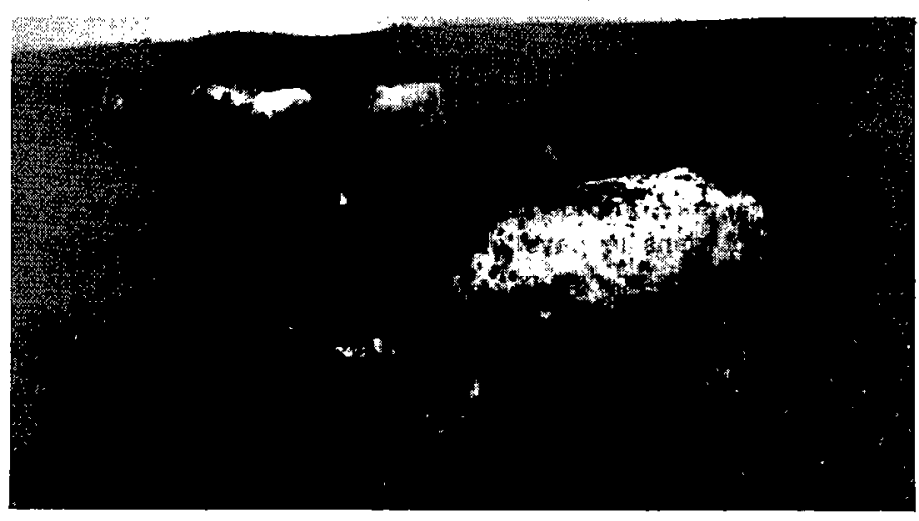

Taureau Mbororo, variété "Akou ".

(Cliché Tobie Schuller
Vaches Mbororo

variété "Akou ", 6 et 7 ans.

(Cliché Tobie Schuller)

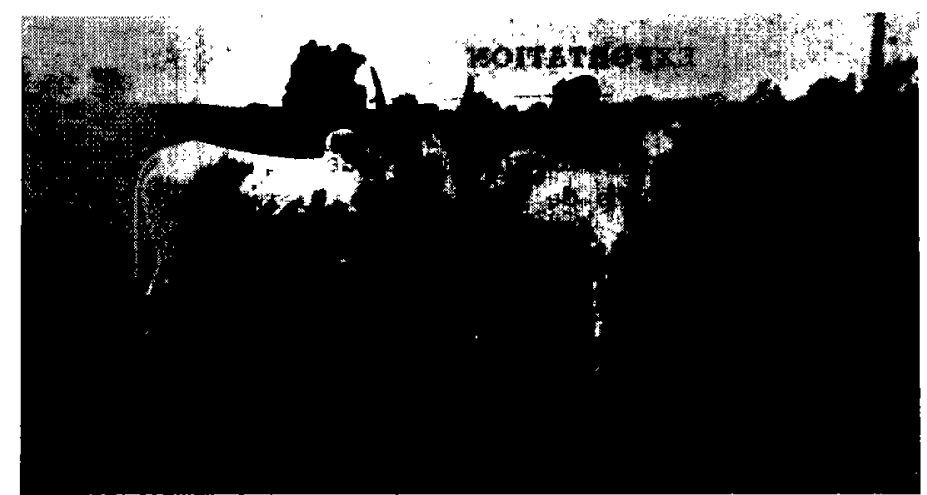




\section{CONSOMMATION LOCALE}

Actuellement en Adamawa, pour une population de 160.000 habitants, il est abattu annuellement plus de 10.000 bovins dont le poids moyen de viande est de 170 à $180 \mathrm{~kg}$, soit en moyenne $11 \mathrm{~kg}$ de viande de boeuf par habitant et par an.

On constate en Adamawa que cette consommation locale de viande s'accroît chaque 'année, au fur et à mesure, bien sûr, que les courants commerciaux se développent dans le pays et créent chez l'autochtone des besoins nouveaux. provenance de l'Adamawa s'élevait en 1932 à 13.000 têtes.

En 1950, il atteignait 33.000 têtes
En 1951, - $36.000-$
En 1952, $\quad-\quad 44.000-$

ce qui représente un volume de transactions considérable dont la valeur moyenne, pour l'année 1952 seulement, peut être chiffrée à plus de 800 millions (huit cent millions) de francs C.F.A.

I'augmentation de ces exportations de bétail sur pied peut être encore poursuivie sans danger en

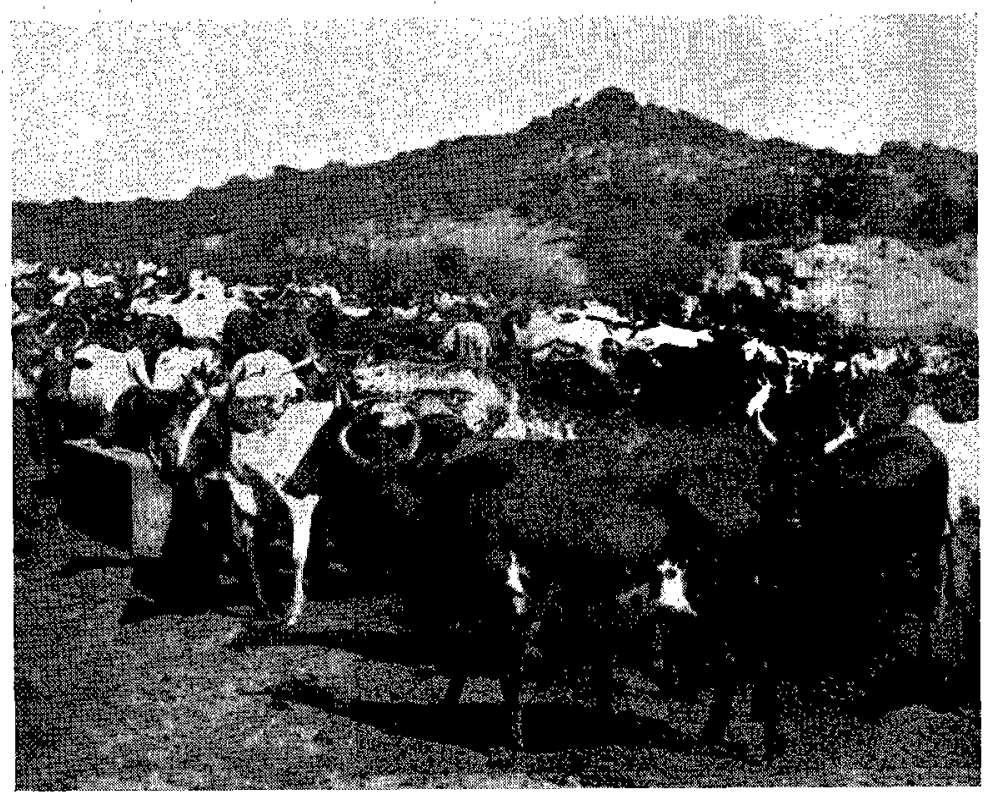

(Cliché Mandon)

Troupeau de zébus peuls à l'abreuvoir d'eau natronée.

\section{EXPORTATION}

a) De bétail sur pied.

C'est par les pistes à bétail aménagées que le plus gros tonnage de bovins de boucherie, en provenance de l'Adamawa, continue d'être acheminé sur les centres consommateurs du Sud, de l'Est et de l'Ouest Cameroun.

La traversée des zones à tsé-tsé se fait sous le couvert de la chimio-prévention, autrefois le bromure de Dimidium, aujourd'hui le chlorure qui, à la dose de $0 \mathrm{mg} 8$ par $\mathrm{kg}$ de poids vif protège efficacement notre bétail de boucherie durant les 800 kilomètres de pistes qu'il doit parcourir des contrées de production animale à la capitale Yaoundé.

Le chiffre des exportations de bétail sur pied en
Adamawa : la disponibilité annuelle en bovins de boucherie étant de 60.000 têtes environ.

b) De viande de bœuf par avion.

Les premiers essais en Adamawa de transport par avion de viande en quartiers datent de 1950. Depuis cette époque, impressionnante a été, à notre avis, l'augmentation du tonnage de viande de bœuf expédié de cette façon sur les D.C. 3 et D.C. 4 cargo des Compagnies aériennes Air-France et U.A.T., de Ngaoundéré vers les centres importants de Douala, Yaoundé, du Moyen-Congo, du Gabon, de la Guinée Espagnole, de Fernando Po et du Congo Belge :

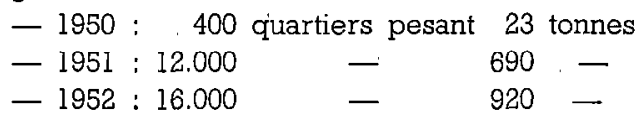


ce qui représente un total de 7.100 bovins ayant donné une moyenne de 230 kilos de viande en quartiers.

De tels rendements peuvent être considérés comme remarquables, puisque obtenus sur des zébus de race locale, non améliorés, entretenus constamment en plein air et nourris uniquement de ce que peut leur fournir le pacage naturel.

Par ailleurs, ce système de transport des viandes camerounaises par voie aérienne s'avère des plus rentables, comme nous l'avons déjà précisé dans une étude comparative sur l'intérêt économique du transport des bovins de l'Adamawa sur pied et par avion, et permet, en outre, de commercialiser rapidement un produit de qualite dans les conditions d'hygiène les meilleures.

C'est pourquoi nos efforts tendent aujourd'hui à intensifier le développement de ce mode d'exportation des ressources en viande de l'Adamawa et parallèlement à doter, dans les délais les plus courts, ses plus importants centres de production animale d'abattoirs frigorifiques modernes.

A mien pas douter, le transport par avion de viandes réfrigérées en provenance de l'Adamawa constitue le plus sûr moyen d'assurer leur réputation commerciale et d'accroître leurs débouchés.

\section{CONCLUSIONS}

Lorsqu'un pays d'altitude, aux conditions climatiques tempérées et constamment à l'abri des grandes enzooties, comme l'est le plateau de l'Adamawa, entretient plus d'un demi-million de bovins de choix sur ses 2.500 .000 hectares de savanes herbeuses où abondent naturellement toute l'année, d'une part, les espèces de graminées les plus diverses et, d'autre part, l'eau ainsi que les sources minérales, la préoccupation dominante du Service Zootechnique qui y est installé, doit être l'amélioration qualitative de ce bétail.

C'est pour réaliser ce programme que la Station Zootechnique de Wakwa, près de Ngaoundéré, a porté ses efforts, de 1935 à 1950 sur les travaux zootechniques suivants :

- Elevage et sélection des zébus de race peule;

- Importation de reproducteurs et reproductrices de race montbéliarde;

- Production sur place de sujels purs montbéliards;

- Amélioration de la race bovine locale par croisement continu avec les sujets montbéliards importés ou nés en Adamawa;

- Distribution dans le milieu africain de reproducteurs métis montbéliards;
- Fixploitation des meilleurs géniteurs métis Montbéliards dans des Centres d'Insémination.

A partir de 1951, après les échecs enregistrés et depuis longtemps prévus ( 1 ) en matière de production en Adamawa de sujets purs et métis près du sang Montbéliard plus particulièrement, la Station Zootechnique de Wakwa estimait que les opérations de croisement pour l'amélioration qualitative du bétail de l'Adamawa ne devaient pouvoir s'effectuer avec succès qu'en utilisant des sujets zébus sélectionnés de race Brahma provenant de l'Amérique du Nord ou du Sud.

Elle passait donc commande au Texas de 10 taureaux, inscrits au Herd Book américain de la race Brahma, qui arrivaient en Adamawa le 24 décembre 1952.

L'introduction au Cameroun de taureaux Brahma est par conséquent de date assez récente. C'est la raison pour laquelle nous n'étudierons que dans un prochain article, d'une part, l'acclimatement de ces reproducteurs Brahma en Afrique Noire ainsi que les modalités techniques de leur exploitation à l'intérieur des troupeaux de reproductrices ZébuPeul sélectionnées que possède déjà la Station de Wakwa, et, d'autre part, les premiers résultats de croisement que nous aurons obtenus.

\section{BIBLIOGRAPHIE}

Encyclopédie de l'Efrique Française (Annóc 1951). Rapport annuel dn gonvernement français sur l'administration du Cameroun (Année 1951).

STRUMPEL Kurt (Capitaine). - Histoire de l'Ádamawa (Année 1912).

Bulletins de la Société d'Études Camerounaises (1935-1937) (1943-1947), suivis de Études Cameronnaises (Centre du Cameroun de l'Institut Français d'Afrique Noire à Douala).

Rapport annuel de la région de l'Aldamawa (1952).

Rapports et communications de la Station Zootechnique de Wakwa (de 1934 à 1952).

(1) Dans notre article « L'Élevage des Bovins et l'Insémination Artificielle en Adamawa "), paru en 1948 dans la Revue d'Élevage et de Médecine Vétérinaire des Pays Tropicaux, nous avions déjà communiqué les résultats obtenus en ce qui concerne l'élevage de P.S. Montbéliards en Adamawa ainsi que les divers croisements pratiqués. Nous ne saurions donc les rappeler ici. 